\section{Understanding the barriers to the utilization of primary health care in a low-income setting: implications for health policy and planning}

\author{
Kurfi Abubakar Muhammed, ${ }^{1}$ \\ Kalu Nnena Umeh, ${ }^{2}$ Sambo M. Nasir, ${ }^{3}$ \\ Idris Hadejia Suleiman ${ }^{3}$
}

${ }^{1}$ National Health Insurance Scheme, North West Zonal Office, Kaduna;

${ }^{2}$ National Health Insurance Scheme Headquarters, Abuja;

${ }^{3}$ Department of Community Medicine, Ahmadu Bello University, Zaria, Nigeria

\section{Abstract}

The essence of primary health care is the provision of essential health services and commodities to individuals and communities using available, acceptable and sustainable resources. However, there has been a growing lack of confidence by the populace as evidenced by poor utilization of the services. This study sought to identify the predominant barriers affecting the utilization of primary health care services in Batsari Local Government in Katsina State, Nigeria. A cluster of 630 households was surveyed in the catchment of the 21 health primary health facilities. A catchment been defined as a household located within $5 \mathrm{~km}$ of a primary health center. Using a three digit randomly generated numbers a household was selected. Once selected the start house and twenty-nine contiguous houses were visited. a total of 630 households were surveyed. In all households, questions were asked on the predominant health problems, as well as the major determinants of access and utilization of primary health care services .The results were computed and analyzed using the Statistical Package for Social Sciences software SPSS. Version 17.0. The findings from all the respondents $(\mathrm{n}=630)$ showed that majority of the people preferred to seek care from the patent medicine stores $(53.63 \%)$ as against only $7.6 \%$ who utilized the primary health care services. The commonest reasons why respondents do not utilize these services were lack of essential drugs, high cost of services as well as inadequate infrastructure in primary healthcare facilities. The study has highlighted some of the multiple factors affecting the utilization of primary healthcare services. It is expected that these findings will guide policy makers in improving healthcare delivery particularly where the need is greatest - at the grassroots - in line with the national health policy and national health strategic development plan.

\section{Introduction}

Primary health care is defined as essential health care that is based on scientifically sound and socially acceptable methods and technology made universally accessible to individuals and families in the community through their full participation and at a cost that the community can afford to maintain at every stage of their development in the spirit of self reliance and self determination. ${ }^{1}$ The fundamental problems of primary health care (PHC) have been recognized and respected by all nations, yet there are divergences and ambiguities in interpretation across countries worldwide. A recent report by WHO African region puts the level of PHC utilization at $5-7 \%$ in the region this translates to about $95 \%$ under-utilization of the services. ${ }^{2}$

The fundamental aim of PHC is to ensure universal access to available resources in order to provide adequate coverage of the most important health needs of the people. Factors that determine the utilization of health care services include geographical and economic accessibility, literacy level and perceived derivable benefits. ${ }^{3}$ Access represents the fit between characteristics and expectations of the providers and clients, these characteristics are actually grouped into the $5 A^{s}$ of access that is accessibility, affordability, availability, accommodation and acceptability. While affordability is an index of how the provider charge the client and how the cost of care relates to the willingness to pay for services, availability denotes the extent to which providers possess the requisite resources, such as personnel and technology to meet the needs of clients; accessibility refers to the geographical dimension of access, which tends to explain how easily the client can physically reach the providers location. Accommodation reflects the extent to which the providers operation is organized in ways that meets the constraints and preferences of the client, of great concern are hours of operation and the ability of patient to access care irrespective of status or prior appointment. Acceptability captures the extent to which the client is comfortable with the nature of services with respect to his culture, religion and other social dimensions. These chains intertwine and aid in determining the utilization or otherwise of any health care services.

The 2009 World Development Report released by the UNDP and the Nigeria National Demographic Health Survey 2008 portray the extent of under-utilization of primary health care services in the nation in general and the Northern part of the country in particular. For example, the overall national immunization coverage is $29 \%$ while Katsina state in the North West (the location of this study) record-
Correspondence: Kurfi Abubakar Muhammed, National Health Insurance Scheme, North West Zonal Office, Kaduna, NIgeria.

Tel. +23.48034534498

E-mail: abukurfi@gmail.com

Key words: primary health, barriers, utilization, health policy and planning.

Acknowledgements: we are grateful to all the household members who participated in this study; we also wish to acknowledge the contribution of all the staffs of the primary health care department of Batsari Local Government area of Katsina State, more specifically those working in the health facilities where this study was carried out.

Contributions: KAM and SMN conceptualized and designed the study protocols; KAM carried out the field study work; KAM and KNU analyzed and interpreted the data; IHS critically reviewed the findings. All authors read and approved the final manuscript.

Conflict of interests: the authors declare no potential conflict of interests.

Received for publication: 2 February 2012.

Revision received: 1 June 2012.

Accepted for publication: 26 May 2013.

This work is licensed under a Creative Commons Attribution NonCommercial 3.0 License (CC BYNC 3.0).

(OCopyright K.A. Muhammed et al., 2013

Licensee PAGEPress, Italy

Journal of Public Health in Africa 2013; 4:e13

doi:10.4081/jphia.2013.e13

ed less than $1 \%$ coverage for all antigens. Again, national contraceptive prevalence rate was $15 \%$ but only $1 \%$ in Katsina. The state had an antenatal coverage of $14.4 \%$ with only $4.7 \%$ of the women delivering in the hospital. ${ }^{4,5}$ Despite the fact that a lot of resources have been dedicated and invested by the different tiers of the government, patients still by-pass the PHC to directly access services in secondary and tertiary health facilities. This results in over-burdened and overstretched facilities with consequent reduction in quality of services at higher levels of care.

The national health policy in Nigeria has been formulated within the context of the health strategy of the New Partnership for Africa's Development, a pledge by African leaders based on a common vision and a firm conviction that they have a pressing duty to eradicate poverty and place their countries individually and collectively on a path of sustainable growth and development. The United Nations Millennium Development Goals (MDGs) to which Nigeria, like other countries, has committed to achieve; and a locally developed eco- 
nomic blue print; the Economic Empowerment and Development Strategy (NEEDS) which is aimed at re-orienting the values of Nigerians, reforming government and institutions, growing the role of the private sector, and enshrining a social charter on human development with the people of Nigeria; as well as the development of a comprehensive health sector reform program as an integral part of the NEEDS. In all aspect of the policy formulation and implementation process primary health care remain the basic philosophy and strategy for national health development. This study aims to highlight critical barriers to the utilization of PHC so as to generate evidencebased information, that will assist policy makers and health management teams understand the challenges of primary healthcare delivery from the viewpoint of the beneficiaries and develop constructive solutions to improve on it.

\section{Materials and Methods}

\section{Batsari: population and primary health care services}

Batsari is a semi-urban area of Katsina state, northwestern Nigeria. With a population of $208,978 .{ }^{5}$ The area is predominantly inhabited by Hausa Fulani Moslems, who are mostly engaged in subsistence farming and cattle rearing as a means of sustenance. The local government area (LGA) secretariat has pipe borne water and electricity but most of the other villages lack these services. The LGA has 21 twenty one functional health facilities; these include a comprehensive health centre, 3 Maternal and Child Health centers', as well as several dispensaries and health outposts. There is a total of 201 technical, administrative and auxiliary staff working in the health department: a cluster of households was surveyed in the catchment of the 21 health facilities. A total of 630 households in the catchment area of each of the facilities was selected, a catchment been defined as a household located within the national health policy guideline of $5 \mathrm{~km}$ walking distance to a primary health center. Using a three digit randomly generated numbers, the start household was selected direction from the PHC was selected, if the direction selected did not lead towards a residential. Once selected the start house and the twenty nine contiguous houses were visited, at the household only the head of the households was interviewed, where the household head declined or is not present, the survey team continued to the nearest adjacent house until the 30 had been selected.

Data were collected by trained community health extension workers using structured questionnaires. Questions asked included demographic characteristics of households, the recent history of illness, preferred places of treatment, ease of access to the services, the distance to the nearest primary health facility, the predominant barriers to the utilization of the services, the respondents' perceptions of the solutions to these barriers, the existence or otherwise of village and ward development committees and whether these committees contribute to the improvement in utilization of PHC services. Issues to do with satisfaction and health service trust were approached by asking the households what services they would recommend to others for treatment of various child hood and adult conditions. The data collected was manually sorted then analyzed using SPSS version 17.0.

\section{Results}

\section{Demographic findings}

In all 630 households, $99.3 \%$ of the household heads were males, with a mean age of 39.8. The average household size was 5.9 with farming being their major occupation. If literacy is the ability to identify, understand, interpret, create, communicate and compute, using printed and written materials then $52.3 \%$ of the population are not literate. ${ }^{6}$ There is an average of 2 health facilities per political ward; this translates to a ratio of 1 facility to about 25,000 people. The pattern of male predominance among the household heads is also replicated in the health sector as only $4.3 \%$ of staff are females. Most of the workers are auxiliary and non skilled with the bulk of them been community health extension workers. There is only 1 medical doctor in the service of the LGA, making the doctor patient ratio to be 1:208,978. The LGA also has 2 qualified midwives in its service, which means a ratio of 1 midwife to 104,489 patients at any given time.

\section{Health services received during the last illness}

The predominant health care problems of the households one month prior to the study were assessed. $27.6 \%$ of the households said fever was the major reason why they sought medical care, while cough and other respiratory tract infections accounted for $24.7 \%$. Other predominant health conditions are diarrheal diseases, malnutrition, ear and eye infections as well as HIV/AIDS.

\section{Services offered by the facilities}

Only $47.5 \%$ of these facilities are within the National Health policy guideline of $5 \mathrm{~km}$ or 30 min walking distance range and only $36.8 \%$ of the facilities operate on 24 h service. Majority of the facilities only offer immunization, health education and general outpatient services. Only a few offer essential obstetrics care (4.1\%) and family planning services (21\%).

\section{Preferred treatment sites}

Most of the respondents preferred the patent medicine vendors and pharmacies $(53.63 \%)$ as the major source of care. Only $7.6 \%$ utilize the PHC centers in the villages; others used traditional medicine (16.7\%) and private health facilities (14\%).

\section{Reasons for non-utilization of the services}

Numerous reasons were given for the non utilization of the PHC facilities but the most common were non availability of essential drugs (27.6\%) closely followed by the high cost of services $(25.9 \%)$, while $15.5 \%$ cited inadequacy of medical staff. Poor community involvement accounted for $9.2 \%$ while the negative attitude of staff was the main reason among $8.0 \%$ only $0.3 \%$ mentioned the absence of a medical doctor as their reason for not utilizing the primary health care facilities.

\section{Perceived solution to the problems}

On the issue of possible solutions to the problems with PHC services, $27.3 \%$ of them suggested essential drugs should be always be stocked while $25.2 \%$ were of the view that the cost of services should be reduced; $21.8 \%$ think recruiting more staff was the best solution to the problem and $8.6 \%$ were of the view that involving the community would enhance the primary health care services utilization. Only $5.7 \%$ were of the opinion that bringing the health facility closer would improve utilization.

\section{Satisfaction with the services}

Satisfaction with the services; With respect to the level of satisfaction with primary health care services assessed using the Liker's 5 point scale, $63.8 \%$ of the population said they were not satisfied and $19.0 \%$ said they were strongly dissatisfied with the services. Only $8.0 \%$ of the population said they were satisfied with the services.

\section{Availability of village and ward development committees}

The availability and functionality of village and ward development committees was also assessed and it was found that only $12.5 \%$ of these committees are functional and actively involved in the day to day affairs of their primary health centers. 


\section{Discussion}

The study found the predominant health problems to be preventable and communicable which is in agreement with the level of development of the area. Similar studies elsewhere have also revealed a similar picture where fever was the leading complaint for most household especially among under fives. ${ }^{7}$ The pattern of ailments in the locality can serve as a proxy indicator of the level of development of the area and also emphasizes the need for more preventive measures than curative ones in tackling the health care challenges in the area.

The study also found that majority of the household prefer to utilize the patent medicine vendors or pharmacies as the first line of therapy, attributing non availability of essential drugs as the predominant barrier to their use of the primary health facility. The preference for patent medicine stores as the first point of contact during ailments is probably an indication that the people utilize health services more for curative rather than preventive reasons. Likewise, the non availability of essential drugs in most of the facilities calls to question the effectiveness of the drug revolving fund in the LGA and the free maternal and neonatal health services recently launched by most northern states in Nigeria.

Studies elsewhere have shown that the utilization of maternal, and childhood services is affected by socio-demographic, household, community and state factors, these factors also affect the indicators of maternal health service utilization by selected localities. ${ }^{7,8}$ Household socio-economic status has also been found to be closely correlated with the use of antenatal services such that the odds of reporting use are almost six times as high among women from the richest households compared to their counterparts from the poorest household especially with respect to attitudes towards family planning and ideal family size. ${ }^{9}$ There are also significant variations in these indicators by place of residence, the level of media saturation, family size as well as the ratio of PHC centers to the population in the state of residence. ${ }^{10,11}$

The doctor-patient ratio of 1:208,978 is far below the WHO accepted standard for low income nations pegged at 1:5000. ${ }^{12,13}$ Statistics indicate that the doctor-patient ratio in Ghana is $1: 13,000$, and in Tanzania the average doctor patient ratio is $1: 23,000$ while nurse patient ratio is $160 / 100,000$ but $6 / 100,000$ in rural districts. ${ }^{14}$ Not only is this high ratio below the globally accepted standard but it is also an indicator of the enormous human resource challenges affecting the health care systems in Nigeria. This has the potential of demoralizing both staff and patients and therefore limits the utilization of these services. The situation in the country has been worsened by the exodus of health professionals, particularly doctors, to seek greener pastures in more developed economies. Every year Africa losses many of its health workers-about 23,000 African health professionals migrate to developed countries every year costing $\$ 4$ billion to replace. ${ }^{15}$

The variations in the skilled man power availability across different zones of Nigeria are also obvious for example; while the SouthWest of the country has about 7,300 doctors the North-East has only $63 .{ }^{16}$ The average primary health center in Kogi state in north central Nigeria has 7.85 health workers, but the average for health posts was only 2.3 workers. ${ }^{17}$ The majority of the health workers in this locality are community health extension workers and the reliance of the countries PHC system on this cadre of staffs with little or no supervision has placed the primary health care system in danger, as it has gradually converted these cadre staffs into potential medical doctors, making them do more harm than good.

Not only are the facilities not widely distributed, they are also not evenly distributed; For example, only $47.5 \%$ of these facilities are within the National Health policy guideline of $5 \mathrm{~km}$ or 30 min walking distance range this implies that $52.4 \%$ of the facilities are not within this range. The distribution of these facilities in areas that have no or few inhabitants lends credit to the belief that most of these centers are built for political and economic purposes and not with the involvement of the communities. These not only limits the rate of utilization but have serious implications for conditions that occur at night especially emergencies.

Juxtaposing the pattern of ailment and the preference for patent medicine vendors and pharmacies over the primary health care facilities is the high rate of traditional medicine utilization $16.9 \%$, this is perhaps attributable to the belief that some ailments e.g. fractures, mental illnesses and convulsions are exclusively the domain of traditional medical care and not orthodox care. In Ghana, Mali, Nigeria and Zambia, herbal medicines are the first line of treatment for $60 \%$ of children with high fever from malaria. ${ }^{18,19}$ About $65 \%$ of births delivered in Nigeria especially the rural areas are conducted by traditional birth attendants and these take place outside the health facility. ${ }^{19}$ In rural Burkina Faso modern health care facilities are only consulted by $19 \%$ of the population, others choose home treatment $52 \%$, traditional healers $17 \%$, or local village health workers 5\%; this translates to a utilization of government services as low as $0.17 \%$ consultations per capita. ${ }^{20}$

In Africa, up to eighty percent of people use traditional medicine for primary care, while in Nigeria as much as $85 \%$ use and consult traditional medical care for health care, social and psychological benefits. ${ }^{21}$ In Layin Zomo community of Zaria, north western Nigeria; patent medicine stores were the major source of treatment for majority of the illnesses, public health facilities were used in by only $31.2 \%$ of the cases while $16.7 \%$ sought treatment from private clinics. Traditional healers were also utilized in $5.2 \%$ of the instances while others did not seek any form of treatment. ${ }^{22}$

\section{Conclusions and Implications}

The study has provided a basis for understanding and appreciating the complex factors affecting the utilization of primary health care services. One of the major weaknesses in the health sector today is the non-existence of important health legislation as well as the out datedness, contradictions and ambiguities of some existing health policies. Periodic studies such as this that exposes some of the major barriers to the use of services from the perspective of the end user could assist in guiding policy formulations and decision making to ensure that primary health care remains the fulcrum of all health policies in Nigeria.

\section{Ethical considerations}

The study protocol was approved by the Ahmadu Bello University Research Ethics Committee and the authority of the LGA PHC department was sought prior to the study. Before commencement of interviews, the objectives of the study and the contents of the questionnaire were explained to each of the subjects by research assistants, and verbal consent for participation obtained. Participants were assured that the data which was gathered would be used only for research purpose.

\section{Limitations of the study}

Only households within $5 \mathrm{~km}$ of the primary health care centers were included, persons living further away from the health facilities may have different perceptions and barriers to the utilization of the services. Respondents may also over rate the services for fear of losing such services or may express satisfaction with the services out of ignorance of what constitutes the ideal. Selecting only households within the vicinity of the of the primary health centers may have meant that low and middle income households are over represented as primary health centers are typically often cited in low income neighborhoods. 


\section{References}

1. Olawiyola LM, Adelaye OA, Rural infrastructural development in Nigeria; Between 1960-1990-problems and challenges. J Social Sci 2005:91-6.

2. National Population Commission (NPC) [Nigeria] and ICF Macro. 2004. Nigeria Demographic and Health Survey. Abuja: National Population Commission and ICF Macro; 2003.

3. National Population Commission (NPC) [Nigeria] and ICF Macro. 2009. Nigeria Demographic and Health Survey. Abuja: National Population Commission and ICF Macro; 2008.

4. World Health Organization. The World Health Report, 2008 - PHC Now More than Ever. Geneva: World Health Organization; 2008.

5. Federal Republic of Nigeria. Nigerian health review - PHC in Nigeria after Alma Ata. Abuja: Federal Republic of Nigeria; 2007.

6. World Health Organization. Information on integrated management of childhood illness at primary health care level - information pack. Geneva: World Health Organization; 2000.

7. Department for International Development (DFID). Primary health care, commodity security: country case study. Abuya: Nigeria Commissioned jointly by The Department for International Development and the Netherlands
Ministry of Foreign Affairs; 2005.

8. Adindu A, Babatunde S. Health managers perception of the primary health care management information system: a case study of Bama local government in Northern Nigeria. Nig J Med 2006;15:266-70.

9. Sen G, Ostlin P, George A. Gender inequity in health: why it exists and how we can change it. Geneva: WHO Commission on the Social Determinants of Health; 2007.

10. . Benjamin CO, Nnaemeka AC. Child schooling in Nigeria: the role of gender in urban, rural, North and South Nigeria. Available from: http:/www.saga. cornell. edu/saga/educconf/okpukpara.pdf Accessed: 16 January 2011.

11. Ronald MA, Allan M, Lu An A, et al. Exploring dimensions of access to medical care. J Public Health 2004;94:264-7.

12. Federal Republic of Nigeria. National Population Census Figures; 2006.

13. Braverman P, Krieger N, Lynch J, 2000. Health inequalities. Health inequalities and social inequalities in health. Bull World Health Organ 2000;78:232-4.

14. World Health Organization. Primary health: a first assessment - people report on primary health care. Geneva: World Health Organization; 1985. pp 6-9.

15. Murray CJL, Gakidou EE, Frenk J, 1999. Health inequalities and social group differences: what should we measure? Bull World Health Organ 1999;77:537-43.

16. Javanparast S, Coveney J, Saikia U. Exploring health stakeholders' perceptions on moving towards comprehensive pri- mary health care to address childhood malnutrition in Iran: a qualitative study. BMC Health Serv Res 2009;9:36.

17. Stella B, Adesegun F. Determinants of use of maternal health services in Nigeria looking beyond individual and household factors. BMC Pregn Childbirth 2009;9:43.

18. Ejembi CL, Alti Muazu M, Chirdan 0, et al. Utilization of maternal health services by rural Hausa women in Zaria environs, northern Nigeria:has primary health care made a difference. J Commun Med Primary Health Care 2004;16:47-54.

19. Sambo MN, Ejembi CL, Adamu YM, Aliyu AA. Out-of-pocket health expenditure for under-five illnesses in a semi-urban community in Northern Nigeria. J Commun Med Primary Health Care 2004;16:29-32.

20. Adeniyi J, Oladepo 0, Soyibo A. Survey of primary health care service delivery in Lagos and Kogi: a field report. Mimeo: African Regional Health Education Center, University of Ibadan; 2003.

21. Department for International Develop ment. Increasing people's access to essential medicines in developing countries: a framework for good practice in the pharmaceutical industry - A UK Government policy paper; 2005. Available from: http://www.aidsportal.org/store/909.pdf Accessed: 8 March 2011.

22. Obionu CN. Primary health care for developing countries. 2nd ed. Abuja: Institute of Development Studies, University of Nigeria; 2007. 